\title{
Home palliative care and end of life issues in glioblastoma multiforme: results and comments from a homogeneous cohort of patients
}

\author{
Alfredo Pompili, M.D., ${ }^{1}$ Stefano Telera, M.D., ${ }^{1}$ Veronica Villani, M.D., Ph.D., ${ }^{2}$ \\ ANd Andrea PACe, M.D.2 \\ Departments of ${ }^{I}$ Neurosurgery and ${ }^{2}$ Neuro-Oncology, "Regina Elena” National Cancer Institute, Roma, Italy
}

\begin{abstract}
Object. Glioblastoma multiforme (GBM) is a rare tumor whose prognosis has remained poor over the years despite innovative radio- and chemotherapies, and important technical advances in neurosurgery such as intraoperative imaging, fluorescence, Cavitron ultrasonic surgical aspirator, and neuronavigation. Particular attention has been dedicated in the last years to the end of life (EOL) period in cancer patients for both ethical and socioeconomic issues. Good palliative care at home avoids improper and expensive hospitalizations, and helps and trains families, caregivers, and patients in facing a difficult situation.

Methods. In 2012-2013 the authors' group cared for 197 patients with brain tumors. Of these there were 122 with GBMs: 64 died and 58 are still receiving assistance. The clinical conditions are periodically evaluated with the following scales: Barthel Index, Karnofsky Performance Scale, and Mini-Mental State Examination. Home care staff includes 2 neurologists, 5 nurses, 2 psychologists, 3 rehabilitation therapists, and 1 social worker. The intensity of care changes at the different stages of disease, ranging from low to medium levels of intensity at the progression stage (more than 1 access weekly) to high levels of intensity at the EOL stage (at least 3 accesses weekly). Control MRI studies are obtained every 3 months before terminal progression.

Results. Overall in this sample of patients there were 2838 home visits and 11,714 days of assistance. Thirty-four patients (14 female and 20 male) died at home (53.1\%); 22 (13 female and 9 male) at the hospice (34.4\%); and 8 (4 female and 4 male) at the hospital (12.5\%). A positive impact on caregivers for home assistance was recorded in $97 \%$ of cases, for nursing in $95 \%$, communication in $90 \%$, rehabilitation at home in $92 \%$, and social work help in $85 \%$. Also, $72 \%$ had an improvement in their quality of life scores due to rehabilitation.

End of life palliative sedation with midazolam was necessary in $11 \%$ of cases to obtain good control of symptoms such as uncontrolled delirium, agitation, death rattle, or refractory seizures. Intramuscular phenobarbital is the authors' drug of choice for the severe seizures that occurred in $30 \%$ of cases. The reduction of steroid dosage is also used to decrease wakefulness. Steroids were withdrawn in $45 \%$ of patients dying at home, mild hydration was done in $87 \%$, and tube feeding in $13 \%$. The decision-making process at the EOL stage is time consuming, but the degree of distress of the family is inversely proportional to the extent of the preparatory period.

Conclusions. A previous paper showed the positive cost-effectiveness of home assistance for a larger group of patients dealing with any kind of malignant brain tumors. The same is enhanced for patients with GBM. This requires a well-trained neuro-oncology team that manages neurological deterioration, clinical complications, rehabilitation, and psychosocial problems with a multidisciplinary approach.
\end{abstract}

(http://thejns.org/doi/abs/10.3171/2014.9.FOCUS14493)

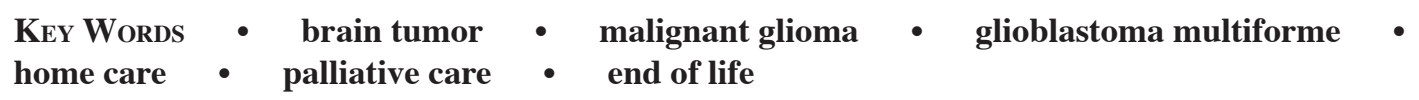

$\mathrm{M}$ ALIGNANT gliomas are rare tumors: the annual incidence in developed countries is 5.8 males and 4.1 females per 100,000 inhabitants. The prognosis remains poor and survival has improved by only a few months over the last 20 years, despite multimodality therapy including surgery, radiotherapy/radiosurgery, and first- and second-line chemotherapy. ${ }^{15}$ Glioblastoma multiforme (GBM) has the worst prognosis: $12-15$ months survival (compared with 2-5 years for anaplastic glioma).

\footnotetext{
Abbreviations used in this paper: $\mathrm{EOL}=$ end of life; $\mathrm{GBM}=$ glioblastoma multiforme; KPS = Karnofsky Performance Scale; $\mathrm{QOL}=$ quality of life.
}

Therefore there is a great need to improve the quality of life (QOL) of both patients and caregivers because all of them have multiple supportive care needs. ${ }^{1}$

Particular attention has been dedicated in the last years to the end of life (EOL) period in cancer patients because of both ethical and economic issues. Good palliative care at home avoids improper and expensive hospitalizations, and helps and trains families, caregivers, and patients to face a difficult situation. ${ }^{6}$ The WHO definition of palliative care is as follows: "palliative care is an approach that improves the QOL of patients and their families facing the problems associated with life-threatening illness, through the prevention and relief of suffering by 
means of early identification and impeccable assessment and treatment of pain and other problems, physical, psychosocial and spiritual."

Supportive care in patients with malignant gliomas includes management of peritumoral edema, venous thromboembolism, seizures, rehabilitation, depression, opportunistic infections, psychological support and communication, and EOL treatments and choices. ${ }^{1,4}$ Patients with brain tumors are quite different from others in the cancer patient population due to the complexity of supportive care needs, the trajectory of disease, the very short life expectancy, and the presence of specific symptoms related to neurological deterioration, and therefore they need a specific palliative approach. ${ }^{12,16}$ Caregivers as well have a high level of distress during the course of the disease and a high need for help in dealing with patients' mental habits and difficult behavior, which change mainly in the last stage of the disease..$^{2,3}$ Strategies to improve continuity of care and decrease the rehospitalization rate are becoming increasingly important in oncology, both to improve the quality of care for cancer patients and to reduce costs for the health care system. ${ }^{11,17}$

Our neuro-oncology group assisted 848 patients with malignant brain tumor at home during the whole duration of the disease in the years 2002-2012. The data demonstrated a very high degree of patient and family satisfaction, and a low incidence of death in hospital (22.2\%) and/ or hospice $(16.8 \%)$ versus death at home $(61 \%)$. A costeffectiveness analysis showed a significant reduction in hospital readmission rates in the last 2 months of life if compared with a home-unassisted control group $(16.7 \%$ vs $38 \%, p<0.001)$ and a significant difference in the cost of care delivered during the hospital stay in the same period ( $€ 7400$ vs $€ 57,000 ; p<0.001)$, according to official data stored in the database of the "Regione Lazio," Italy, Regional Public Health Agency. These last numbers were taken from a series of 72 patients with GBM discharged from our neurosurgical department between January and December 2006 and compared with those of another main hospital in Rome. ${ }^{7-9}$

The comprehensive aim of this assistance is to allow the patients' death at home, to educate families and caregivers, and to avoid improper rehospitalization. The use of the hospice as place to die is necessary when the compliance of the family and/or caregiver decreases, and is offered and facilitated by the staff. The aim of the present study was to retrospectively analyze the data from a more recent and homogeneous group of patients with GBM from the last 2 years.

\section{Methods}

In all of 2012 and 8 months of 2013, our group took in charge 197 patients with brain tumors. Of these there were 122 patients with GBM: 64 died and 58 are still receiving assistance. In the last 4 months of 2013 the study had no financial support, and so that period is not included in this series. In April 2014 the activity started again, with new funding by the Regional Health System. Due to the present financial support and political decisions we will permanently be able to go further with this program.

Our home assistance includes active support in the postoperative period and throughout the course of the disease, modulated according to clinical conditions. Due to obvious organizational and economic problems, assistance is offered to all patients living inside the "Great Highway Ring" of the Rome metropolitan area. None of our patients refused to be enrolled.

Home assistance includes neurological visits and rehabilitation, psychological support for patients and families, and nursing assistance (2 neurologists, 5 nurses, 2 psychologists, 3 rehabilitation therapists, and 1 social worker). In the late stages of disease the intensity of assistance increases and the home care activity reaches a level of "hospice at home."

The patients' clinical condition is periodically evaluated using the following scales: Barthel Index, Karnofsky Performance Scale (KPS), and Mini-Mental State Examination.

The management of patients at home is discussed by the staff in weekly meetings, and is coordinated by the Neuro-Oncological Centre at the "Regina Elena" National Cancer Institute. Home care interventions are patient centered and differ according to the patients' and families' care needs in the different stages of disease. The intensity of care changes according to the appropriate needs at the different stages of disease, ranging from low intensity (weekly home access or contact by phone, standard ambulatory consultation) to a medium level of intensity in patients with disease in the progressive stage (more than 1 weekly access, nursing assistance, psychological assistance, palliative advance care planning) and a high level of intensity of care in the EOL stage (at least 3 weekly accesses). The personalized intervention plan also involves the General Practitioner and Health District Services to ensure 24-hour coverage of the patients. The perception of the quality of care as reported by the caregiver is measured monthly, with a self-administered "customer satisfaction" questionnaire.

Each case is assigned to a dedicated member of the care team as case manager for the identification of care needs and early evaluation of clinical modifications and emerging problems. The case manager is usually a nurse who is supervised by the neurologist assigned to the patient. Every new case is presented and discussed in the weekly home care team meeting. The weekly meeting is also dedicated to the discussion of critical cases, particularly patients at the EOL stage. The team enrolls patients with GBM immediately after the first observation or discharge after surgery. A control MRI is obtained every 3 months, in both patients who receive treatment and in those who are in active observation after that. Patients do not undergo any imaging procedures while in preterminal progression.

\section{Results}

Of the 122 patients who received assistance, 64 died, with a median survival of 13.34 months. The others are still alive and receiving assistance.

\section{Place of Death}

Thirty-four patients (14 female and 20 male) died at home (53.1\%); 22 (13 female and 9 male) in a hos- 


\section{Palliative care and end of life in patients with glioblastoma}

pice $(34.4 \%)$; and 8 (4 female and 4 male) in the hospital $(12.5 \%)$. Overall, in this sample of patients there were 2838 home visits and 11,714 days of assistance. The duration of each home access by one or more members of the staff is at least 50 minutes.

We divided the patients into 2 groups: $>65$ and $\leq$ 65 years, and found that there were some differences regarding the place of death. In the group of patients $>65$ years (29 deceased), 9 male and 4 female patients died at home, 2 male and 7 female patients in the hospice, and 4 male and 3 female patients in the hospital. In the group of patients $\leq 65$ years (35 patients deceased), 11 male and 10 female patients died at home; 7 male and 6 female patients in the hospice; and 1 female patient in the hospital.

\section{Caregiver Satisfaction}

The satisfaction data, collected by the psychologist and the social worker, indicate a positive psychosocial impact on caregivers: for home assistance in $97 \%$ of cases, nursing in $95 \%$, communication in $90 \%$, rehabilitation at home in $92 \%$, and social work help in $85 \%$. A crucial issue in positively influencing the QOL of patients is the caregivers' education: mobilization from bed to wheelchair, adequate nutrition to avoid dysphagia, proper respiratory exercises, proper leg position and exercises to avoid deep venous thrombosis, and prevention of bedsores. The more the caregiver is involved, the better the satisfaction rate.

\section{Rehabilitation Therapy}

After 3 months of home treatment the Barthel Index score had improved in $43 \%$ of the patients, and $72 \%$ also had an improvement in their QOL scores in at least 1 item if compared with their baseline scores (mainly KPS). Patients' self-perception of QOL was no longer measured in the last weeks of life due to cognitive impairment. For evaluation in this phase, the aforementioned caregiver's "customer satisfaction" along with the place of death and number of useless rehospitalizations are crucial.

\section{End of Life Observations and Treatment Options}

One of the purposes of a home care palliative program is to give patients and families adequate relief from suffering - avoiding prolongation of agony - together with psychological and spiritual support. ${ }^{13,14}$ The symptoms in the last month of life may be devastating: epilepsy (30\%), headache $(36 \%)$, drowsiness $(85 \%)$, dysphagia $(85 \%)$, death rattle (that is, terminal respiratory secretions; $12 \%$ ), and agitation and delirium (15\%).

Seizures must be treated because they are the most frequent reason for rehospitalization, with QOL worsening and increase of the overall costs. Antiepileptic drugs may be difficult to give to patients with dysphagia, with other ways needing to be found: rectal, intramuscular, sublingual, transdermal, or subcutaneous. In this phase intramuscular phenobarbital is the most useful. Intravenous administration of other antiepileptic drugs may be difficult to manage for the caregivers. ${ }^{10}$

Dysphagia is frequently the first symptom of the EOL period; it occurs approximately 4-5 weeks before death and worsens over days. Its management requires good training of the caregiver(s). At its onset, a discussion should be undertaken with the staff and the family about EOL issues and the necessary or unnecessary treatments as well as nutrition and hydration.

The options about EOL treatments mainly concern "no-treatment" decisions: withdrawal of supportive treatment (steroids, anticonvulsants), and withdrawing or withholding of artificial nutrition and/or hydration in patients with prolonged vegetative state. In our patients, pharmacological palliative sedation was necessary in $11 \%$ of cases to obtain good management of symptoms such as uncontrolled delirium, agitation, and death rattle or refractory seizures. Midazolam is our drug of choice: $5 \mathrm{mg}$, which can be repeated 3-4 times a day or more, and delivered either intravenously, rectally, or submucosally.

Reduction of the steroid dosage may be enough in many cases to attain the required reduction of wakefulness, either because steroids may induce agitation or because there is no more therapy for brain edema. Steroids were withdrawn in $45 \%$ of patients dying at home; mild hydration was given in $87 \%$, and tube feeding in $13 \%$.

\section{Ethical Concerns}

The behaviors of all those involved should be planned by shared decisions from the beginning of home care..$^{10,17}$ This plan was not always easy to set up, and we do not have a well-defined guideline to suggest. Patients with malignant brain tumor frequently have cognitive problems that may affect their competence to express preferences. We recorded patients' advance directives in only $6 \%$ of the cases. According to recent studies, early organization of Advance Care Planning could contribute to improving EOL decision making. ${ }^{17}$ The decision-making process is time consuming but the degree of distress in the family is inversely proportional to the extent of previous preparation in each case.

To our knowledge, the first paper about the caregiver's burden in neurosurgery was published in the early 1990s about caregivers of patients who had undergone operations for complex cranial base meningiomas. ${ }^{5}$ More recent papers report on the burden of caregivers of patients with malignant brain tumors., ${ }^{2,3}$ The suffering and despair of the family members who are caring for the patient is often neglected, suggesting the usefulness of a more global approach to the care of the whole affected family. The role of caregivers is of paramount importance in a home care situation, also to avoid improper hospitalization in case of acute symptoms. We did not observe any difference in families' or caregivers' behavior based on cultural or social attitudes.

\section{Discussion}

As in other reports, we do not have results from a randomized trial, but our data suggest and demonstrate the efficacy of this kind of assistance in patients with GBM. ${ }^{13,14}$ The cost-effectiveness has been shown in a larger group of patients reported in a previous paper dealing with all patients with malignant brain tumor. ${ }^{7}$

Home care models have been proposed in different countries for cancer patients. ${ }^{6,11}$ There are very few studies specifically addressing palliative care and EOL issues 
in patients with GBM. ${ }^{14,16}$ Better knowledge of clinical, social, and ethical aspects could improve the training and quality of neuro-oncology units.

Nevertheless, some comments about this series may be made. A GBM is different from other malignant brain tumors. It is more aggressive, and the psychological impact of the first months of therapy (surgery and then radio- and chemotherapy according to the Stupp protocol) may be hard to bear. ${ }^{15}$ Time to progression is short after the completion of the first therapeutic approach, and does not leave families enough time to cope with the new situation, although most of the patients have a good QOL (KPS score > 70). This illness, because it is more rare and unexpected than other cancers, is difficult to accept for both patients (when aware) and families.

Italian familial ties are still rather strong, despite the changes taking place in these last decades, whereas in other countries children and relatives may live far away from a patient's home. This fact simplified our approach, which may be more difficult and expensive in a different situation.

Although not statistically significant in this group of patients, the role of female caregivers is predominant if paired with males. Analyzing our data concerning the place of death, the number of men in the group of patients $>65$ years old who died at home is larger than for women in that group. In our society the burden of the care of illness in a family is still mainly on women's shoulders.

\section{Conclusions}

The complex needs of patients with advanced malignant brain tumors - namely GBM-require the management of neurological deterioration, clinical complications, rehabilitation, and psychosocial problems by a well-trained neuro-oncology team with a multidisciplinary approach. Future clinical research strategies should include new models of care for patients with brain tumors, with special attention given to palliative home care models. Palliative programs and home care models of assistance may represent an alternative to in-hospital care for the management of symptoms in patients dying from a brain tumor and may improve the overall quality of care, either for patients or for caregivers, especially in the last stage of disease.

\section{Acknowledgment}

We thank Mrs. Marzia Piccoli, who constantly reviews and organizes the work of the home care staff.

\section{Disclosure}

The program of Home Palliative Care for Brain Tumor is financially supported by the "Regione Lazio" Public Health System.

Author contributions to the study and manuscript preparation include the following. Conception and design: Pompili. Acquisition of data: Villani. Analysis and interpretation of data: Pompili, Villani. Drafting the article: Pompili. Critically revising the article: Pompili, Telera, Pace. Reviewed submitted version of manuscript: Pompili, Telera, Pace. Approved the final version of the manuscript on behalf of all authors: Pompili. Statistical analysis: Pompili.

\section{References}

1. Catt S, Chalmers A, Fallowfield L: Psychosocial and support- ive-care needs in high-grade glioma. Lancet Oncol 9:884891,2008

2. Finocchiaro CY, Petruzzi A, Lamperti E, Botturi A, Gaviani P, Silvani A, et al: The burden of brain tumor: a single-institution study on psychological patterns in caregivers. J Neurooncol 107:175-181, 2012

3. Flechl B, Ackerl M, Sax C, Oberndorfer S, Calabek B, Sizoo $\mathrm{E}$, et al: The caregivers' perspective on the end-of-life phase of glioblastoma patients. J Neurooncol 112:403-411, 2013

4. Ford E, Catt S, Chalmers A, Fallowfield L: Systematic review of supportive care needs in patients with primary malignant brain tumors. Neuro Oncol 14:392-404, 2012

5. Lang DA, Neil-Dwyer G, Garfield J: Outcome after complex neurosurgery: the caregiver's burden is forgotten. J Neurosurg 91:359-363, 1999

6. Ostgathe C, Gaertner J, Kotterba M, Klein S, Lindena G, Nauck $\mathrm{F}$, et al: Differential palliative care issues in patients with primary and secondary brain tumours. Support Care Cancer 18: $1157-1163,2010$

7. Pace A, Di Lorenzo C, Capon A, Villani V, Benincasa D, Guariglia L, et al: Quality of care and rehospitalization rate in the last stage of disease in brain tumor patients assisted at home: a cost effectiveness study. J Palliat Med 15:225-227, 2012

8. Pace A, Di Lorenzo C, Guariglia L, Jandolo B, Carapella CM, Pompili A: End of life issues in brain tumor patients. J Neurooncol 91:39-43, 2009 (Erratum in $\mathbf{J}$ Neurooncol 91:45, 2009)

9. Pace A, Parisi C, Di Lelio M, Zizzari A, Petreri G, Giovannelli M, et al: Home rehabilitation for brain tumor patients. J Exp Clin Cancer Res 26:297-300, 2007

10. Pace A, Villani V, Di Lorenzo C, Guariglia L, Maschio M, Pompili A, et al: Epilepsy in the end-of-life phase in patients with high-grade gliomas. J Neurooncol 111:83-86, 2013

11. Santiago-Palma J, Payne R: Palliative care and rehabilitation. Cancer 92 (4 Suppl):1049-1052, 2001

12. Sizoo EM, Braam L, Postma TJ, Pasman HR, Heimans JJ, Klein $\mathrm{M}$, et al: Symptoms and problems in the end-of-life phase of high-grade glioma patients. Neuro Oncol 12:1162-1166, 2010

13. Sizoo EM, Pasman HR, Buttolo J, Heimans JJ, Klein M, Deliens L, et al: Decision-making in the end-of-life phase of high-grade glioma patients. Eur J Cancer 48:226-232, 2012

14. Sizoo EM, Taphoorn MJ, Uitdehaag B, Heimans JJ, Deliens L, Reijneveld JC, et al: The end-of-life phase of high-grade glioma patients: dying with dignity? Oncologist 18:198-203, 2013

15. Stupp R, Hegi ME, Mason WP, van den Bent MJ, Taphoorn MJ, Janzer RC, et al: Effects of radiotherapy with concomitant and adjuvant temozolomide versus radiotherapy alone on survival in glioblastoma in a randomised phase III study: 5-year analysis of the EORTC-NCIC trial. Lancet Oncol 10:459466, 2009

16. Sundararajan V, Bohensky MA, Moore G, Brand CA, Lethborg C, Gold M, et al: Mapping the patterns of care, the receipt of palliative care and the site of death for patients with malignant glioma. J Neurooncol 116:119-126, 2014

17. Temel JS, Greer JA, Muzikansky A, Gallagher ER, Admane S, Jackson VA, et al: Early palliative care for patients with metastatic non-small-cell lung cancer. N Engl J Med 363:733742,2010

\footnotetext{
Manuscript submitted August 14, 2014.
}

Accepted September 22, 2014.

Please include this information when citing this paper: DOI: 10.3171/2014.9.FOCUS14493.

Address correspondence to: Alfredo Pompili, M.D., Department of Neurosurgery, "Regina Elena" National Cancer Institute, Via Elio Chianesi 53,00144 Roma, Italy. email: pompili@ifo.it. 